\title{
Gravitational and magnetosonic waves in gamma-ray bursts
}

\author{
J. Moortgat and J. Kuijpers
}

\author{
Department of Astrophysics, University of Nijmegen, PO Box 9010, 6500 GL Nijmegen, The Netherlands \\ e-mail: kuijpers@astro.kun.nl
}

Received 12 November 2002 / Accepted 29 January 2003

\begin{abstract}
One of the possible sources of gamma-ray bursts (GRBs) are merging, compact neutronstar binaries. More than $90 \%$ of the binding energy of such a binary is released in the form of gravitational waves (GWs) in the last few seconds of the spiral-in phase before the formation of a black hole. In this article we investigate whether a fraction of this GW energy is transferred to magnetohydrodynamic waves in the magnetized plasma wind around the binary. Using the $3+1$ orthonormal tedrad formalism, we study the propagation of a monochromatic, plane fronted, linearly polarized GW perpendicular to the ambient magnetic field in an ultra-relativistic wind, first in the comoving and then in the observer frame. A closed set of general relativistic magnetohydrodynamic (GRM) equations is derived in the form of conservation laws for electric charge, matter energy, momentum and magnetic energy densities. We linearize the GRM equations under the action of a monochromatic $\mathrm{GW}$, which acts as a driver and find that fast magneto-acoustic waves grow, with amplitudes proportional to the GW amplitude and frequency and the strength of the background magnetic field.
\end{abstract}

Key words. gravitational waves - plasmas - gamma ray: bursts - stars: pulsars: general magnetohydrodynamics (MHD)

\section{Introduction}

The coupling between gravitational waves and electromagnetic waves (EMWs) in a magnetized vacuum has been investigated extensively over the past 40 years by a number of authors (Gertsenshtein 1961; Lupanov 1967; Boccaletti et al. 1970; Zel'dovich 1973; Gerlach 1974). These studies demonstrate the coherent excitation of EMWs by a monochromatic GW propagating perpendicularly to a background magnetic field.

The first calculations including the presence of a plasma were done by Macedo \& Nelson (1983), who found a coupling of GWs to ordinary and extraordinary EMWs, whereas Brodin \& Marklund (1999) derived the parametric excitation of Langmuir waves by a GW propagating through unmagnetized plasma. In Marklund et al. (2000); Brodin et al. (2001a), the authors adopt the $3+1$ tedrad formalism (Thorne et al. 1986; Ellis \& van Elst 1999) and show that the dispersion relation in a tenuous plasma differs only from the vacuum solution by a small wavenumber shift. In a plasma, however, subsequent non-linear conversions such as harmonic generation, might allow the EMW energy to escape as radiation with frequencies high enough to overcome the interstellar plasma frequency (Brodin et al. 2001b). A numerical estimate for the case of a merging NS-NS binary shows that the amplitude of the EMWs can be significant. Longitudinal waves are excited by

Send offprint requests to: J. Moortgat,

e-mail: moortgat@astro.kun.nl higher-order GW-EMW interactions (Brodin et al. 2000) and magnetosonic waves (MSWs) by GWs propagating in a low- $\beta$ plasma (Papadopoulos et al. 2001).

In this article we consider NS-NS mergers as a source for GRBs and apply the $3+1$ formalism to the interaction of the GWs emitted by the merger with the ultra-relativistic wind of magnetized plasma around the binary. In the last seconds before the collapse to a black hole, a considerable fraction of $M_{\odot} c^{2}$ is released into this plasma in the form of GWs (see Janka et al. 2002, Table 1). We show that these waves distort the extremely strong magnetic field, frozen into the plasma, and excite growing magnetosonic waves in the wind. Already before the merger, the binary is embedded in a relativistically expanding magnetized wind of (mainly) leptons from the orbiting neutronstars, so even a small transfer of GW energy to the wind may provide an interesting central engine mechanism to fuel a GRB fireball.

The outline of this article is as follows. In Sect. 2, the covariant expressions for the electromagnetic fields, the energymomentum densities and the orthonormal tedrad for a linearly polarized GW are recapitulated. A closed set of linearized GRM equations in the metric of a GW is derived in Sect. 3 and solved, first in the comoving frame of the plasma (Sect. 4) and then in the frame of an observer at rest with respect to the merger (Sect. 5). A numerical example and the interpretation of our results are given in Sects. 6 and 7, respectively. Sect. 8 comprises our conclusions. 
Throughout Sects. 2-5, Gaussian geometrized units are adopted $(c=1)$ and Latin indices stand for 0, 1, 2, 3. In Sect. 6, however, the numerical results are given in SI units.

\section{Covariant fluid equations}

\subsection{Electromagnetic field equations}

Maxwell's equations in terms of the electromagnetic field tensors and the 4-current density $j_{\mathrm{m}}^{b}=(\tau, \boldsymbol{j})$ are:

$\nabla_{b} F^{a b}=4 \pi j_{\mathrm{m}}^{a} \quad$ and $\quad \nabla_{b} \mathcal{F}^{a b}=0$,

where the covariant Faraday tensor $F^{a b}$ and its dual $\mathcal{F}^{a b}$, can be decomposed into 4-vectors that, in the rest frame of an observer with 4-velocity $u^{a}$, reduce to the electric and magnetic field strengths, $E^{a}=(0, \boldsymbol{E})$ and $B^{a}=(0, \boldsymbol{B})($ Lichnerowicz 1967):

$$
\begin{array}{lll}
F^{a b}=u^{a} E^{b}-u^{b} E^{a}+\epsilon^{a b c d} B_{c} u_{d}, & \mathcal{F}^{a b} \equiv \frac{1}{2} \epsilon^{a b c d} F_{c d}, \\
E^{a} \equiv F^{a b} u_{b} & , B^{a} \equiv \mathcal{F}^{a b} u_{b} .
\end{array}
$$

In ideal MHD, the electric field vanishes in the rest frame of the plasma: $\boldsymbol{E}=0$. Therefore $E^{a} \equiv F^{a b} u_{b}=0$ in any frame and Faraday's tensor reduces to: $F^{a b}=\epsilon^{a b c d} B_{c} u_{d}$.

\subsection{Energy and momentum conservation}

Energy and momentum conservation follow from the 4derivative of the energy-momentum tensor. For a magnetized ideal plasma this can be expressed in terms of the proper matter energy density $\mu$, the pressure $p$ and the metric $g^{a b}$, as (Weinberg 1972; Hawking \& Ellis 1973):

$$
\begin{aligned}
& T^{a b}=(\mu+p) u^{a} u^{b}+p g^{a b}+\frac{1}{4 \pi}\left(F_{c}^{a} F^{b c}-\frac{1}{4} g^{a b} F^{c d} F_{c d}\right) \\
& \nabla_{b} T^{a b}=\nabla_{b}\left[(\mu+p) u^{a} u^{b}+p g^{a b}\right]-F^{a b} j_{\mathrm{m} b}=0,
\end{aligned}
$$

where the current density satisfies Eq. (1).

\subsection{Tedrad system for a gravitational wave}

In the linearized theory of gravity one usually splits the metric: $g^{a b}=\eta^{a b}+h^{a b}$, where $\eta^{a b}=\operatorname{diag}(-1,1,1,1)$ is the Minkowski metric, and $h^{a b}$ is the space-time perturbation caused by the GW. For a transverse-traceless, linearly (+) polarized, monochromatic GW with frequency $\omega_{\mathrm{g}}=k_{\mathrm{g}}$ propagating in the $z$-direction, this field satisfies: $h^{a b}=\operatorname{diag}(0, h,-h, 0)$, with $h(z-t)=h \mathrm{e}^{i \omega_{\mathrm{g}}(z-t)}$ (Misner et al. 1973).

The proper (observer) reference frame, however, comoving with freely moving bodies is defined with respect to the natural orthonormal tedrad (Marklund et al. 2000):

$$
\begin{aligned}
& e_{(0)}{ }^{a}=\left(\partial_{t}, 0,0,0\right) \quad, e_{(1)}^{a}=\left(0,\left(1-\frac{h}{2}\right) \partial_{x}, 0,0\right), \\
& e_{(2)}{ }^{a}=\left(0,0,\left(1+\frac{h}{2}\right) \partial_{y}, 0\right), e_{(3)}^{a}=\left(0,0,0, \partial_{z}\right) .
\end{aligned}
$$

Decomposed with respect to this tedrad, the metric reduces to that of flat space: $g^{(a b)}=\eta^{a b}$ and the GRM equations closely resemble their Newtonian equivalents.

\section{Magnetohydrodynamics in the comoving frame}

The physical situation we want to consider is that of a perfectly conducting, ideal plasma in the presence of a background magnetic field along the $x$-axis, perpendicular to the direction of GW propagation (Fig. 1). First we study the plasma rest-frame where: $\boldsymbol{B}^{(0)}=B_{0} \boldsymbol{e}_{x}, \mu^{(0)}=\rho$ and $\boldsymbol{v}^{(0)}=\tau^{(0)}=p^{(0)}=\boldsymbol{j}_{\mathrm{m}}^{(0)}=\boldsymbol{E}^{(0)}=0$.

The effect of the $\mathrm{GW}$ is to induce small perturbations in all these quantities. Therefore, all equations will be linearized around the unperturbed state.

\subsection{Maxwell's equations}

The relevant, linearized Maxwell equations in the specified tedrad are (Marklund et al. 2000):

$$
\begin{aligned}
& \nabla \times \boldsymbol{B}^{(1)}-\frac{\partial \boldsymbol{E}^{(1)}}{\partial t}=4 \pi j_{\mathrm{m}}^{(1)}+\boldsymbol{j}_{E}^{(1)}, \\
& \nabla \times \boldsymbol{E}^{(1)}+\frac{\partial \boldsymbol{B}^{(1)}}{\partial t}=-\boldsymbol{j}_{B}^{(1)},
\end{aligned}
$$

where the gravitationally induced current densities are just the collected Ricci rotation coefficients or GW-terms:

$\boldsymbol{j}_{\mathrm{E}}^{(1)} \equiv-\frac{B_{0}}{2} \frac{\partial h}{\partial z}^{(1)} \boldsymbol{e}_{y} \quad$ and $\quad \boldsymbol{j}_{\mathrm{B}}^{(1)} \equiv-\frac{B_{0}}{2}{\frac{\partial h^{(1)}}{\partial t}}^{\boldsymbol{e}_{x}}$.

The electric field can be eliminated by assuming the ideal MHD approximation of a collisionless plasma (zero resistivity), where the electric field vanishes in the comoving frame and Ohm's law reduces to:

$\boldsymbol{E}^{(1)}=-\boldsymbol{v}^{(1)} \times \boldsymbol{B}^{(0)}$.

\subsection{Conservation equations}

Charge continuity follows readily from the antisymmetry of $F^{a b}$ and Eq. (1): $\nabla_{a}\left(\nabla_{b} F^{a b}\right)=4 \pi \nabla_{a} j_{\mathrm{m}}^{a}=0$.

The evolution of the magnetic energy $W$ and the Poynting flux $\boldsymbol{S}$ are just projections of Eqs. (4), (5) onto $\boldsymbol{B}^{(0)}$ :

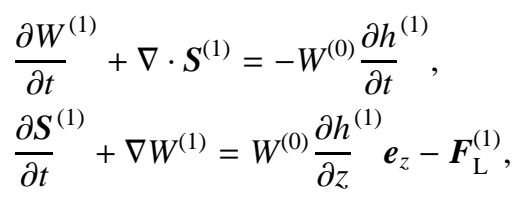

with $\boldsymbol{F}_{\mathrm{L}}=\boldsymbol{j} \times \boldsymbol{B}$ the Lorentz force, $W^{(0)}=B_{0}^{2} / 8 \pi$ and:

$W^{(1)}=\frac{\boldsymbol{B}^{(0)} \cdot \boldsymbol{B}^{(1)}}{4 \pi}, \boldsymbol{S}^{(1)}=\frac{\boldsymbol{E}^{(1)} \times \boldsymbol{B}^{(0)}}{4 \pi}, \boldsymbol{F}_{\mathrm{L}}^{(1)}=j_{\mathrm{m}}^{(1)} \times \boldsymbol{B}^{(0)}$.

Equation (3) results in particle and momentum conservation in terms of the momentum, $\boldsymbol{\pi}^{(1)}=\mu^{(0)} \boldsymbol{v}^{(1)}$ and the spatial stress tensor $\mathrm{T}^{(1)}$ :

$\frac{\partial \mu}{\partial t}^{(1)}+\nabla \cdot \pi^{(1)}=0,{\frac{\partial \pi^{(1)}}{\partial t}}^{+\nabla \cdot \mathrm{T}^{(1)}}=0$,

or, equivalently, as a linearized equation of motion (EOM):

$\mu^{(0)}{\frac{\partial v^{(1)}}{\partial t}}^{(1)} c_{\mathrm{s}}^{2} \nabla \mu^{(1)}=\boldsymbol{F}_{\mathrm{L}}^{(1)}$, 
in terms of the sound velocity $c_{\mathrm{s}}^{2} \equiv \Gamma\left(p^{(1)} / \mu^{(1)}\right)$, where $\Gamma$ is the polytropic index. To first order, the EOM does not contain any GW terms and the coupling of the GW to the plasma occurs only through the Lorentz force as can be seen from Eqs. (9), (11) (or equivalently, through the current density which couples the EOM to Maxwell's Eqs. (4), (5)).

\section{Wave solutions in the comoving frame}

\subsection{Laplace transforms}

The proper way to handle the unstable response of a plasma to a disturbance is to make use of Laplace transforms (Landau \& Lifshitz 1975; Melrose 1986). By giving the frequency or wavenumber a small positive imaginary part, one allows for damping or growth and a way to deal with singularities due to resonance. In an initial value problem it is customary to Laplace transform with respect to time, whereas in a boundary value problem, as we are considering here, a Laplace transform with respect to space is more suitable. These are defined by:

$$
F(s) \equiv \int_{0}^{\infty} \mathrm{e}^{-s z} f(z) \mathrm{d} z, f(z) \equiv \int_{-\mathrm{i} \infty+\eta}^{\mathrm{i} \infty+\eta} \mathrm{e}^{s z} F(s) \mathrm{d} s
$$

where $\eta$ is an arbitrary positive constant chosen so that the contour of integration lies to the right side of all singularities in $F(s)$. Below, we will transform with respect to $s \equiv \mathrm{i} k$, where $\mathfrak{R}[k] \approx k_{\mathrm{g}}=\omega_{\mathrm{g}}$, so the contour of integration follows the real axis except for the poles at $k= \pm \omega_{\mathrm{g}} / u_{\mathrm{A}}$ and $k=\omega_{\mathrm{g}}$, where it deviates infinitesimally into the upper half imaginary plane.

For the time dependence we use Fourier transforms, which implies that the perturbations oscillate at the frequency of the driving GW: $\propto \exp \left(-\mathrm{i} \omega_{\mathrm{g}} t\right)$.

\subsection{Dispersion relations and wave solutions}

We will assume that the tenuous, strongly magnetized leptoid surrounding the binary has a low plasma-beta $\beta_{\mathrm{pl}}=\left(c_{\mathrm{s}} / v_{\mathrm{A}}\right)^{2}=$ $4 \pi p / B_{0}^{2} \ll 1$, where the gas pressure is negligible with respect to the magnetic pressure. The classical Alfvén speed $v_{\mathrm{A}}^{2} \equiv B_{0}^{2} /(4 \pi \rho) \gg 1$ so the displacement current is important. Perturbations propagate in the relativistic plasma with the generalized Alfvén speed, $1 / u_{\mathrm{A}}^{2} \equiv 1+1 / v_{\mathrm{A}}^{2}$.

In Laplace and Fourier space the 13 GRM equations derived in Sect. 3.2 reduce to an algebraic system with 5 non-trivial solutions ${ }^{1}$ :

$v_{z}(\omega, k)=\frac{\omega}{k} \frac{\mu(\omega, k)}{\rho}=\frac{B_{0} j_{y}(\omega, k)}{\mathrm{i} \omega \rho}=\frac{(\omega+k) u_{\mathrm{A}}^{2}}{\omega+k u_{\mathrm{A}}^{2}} \frac{B_{x}(\omega, k)}{B_{0}}$

$=-\frac{E_{y}(\omega, k)}{B_{0}}=\frac{\mathrm{i} h \omega}{2} \frac{u_{\mathrm{A}}^{2}}{\omega^{2}-k^{2} u_{\mathrm{A}}^{2}} \frac{\omega+k}{\omega-k} \delta\left(\omega-\omega_{\mathrm{g}}\right)$.

\footnotetext{
${ }^{1}$ Since the only zeroth order quantities are $B_{0}$ and $\rho$, the superscripts indicating the perturbations will be omitted.
}

The inverse transformations lead to:

$$
\begin{aligned}
& \frac{E_{y}(z, t)}{u_{\mathrm{A}} B_{0}}= \\
& -\frac{v_{z}(z, t)}{u_{\mathrm{A}}}=\zeta \mathfrak{R}\left[\mathrm{e}^{\mathrm{i} k\left(z-u_{\mathrm{A}} t\right)}\left\{1-\zeta_{1} \mathrm{e}^{-\mathrm{i} \Delta k z}-\zeta_{2} \mathrm{e}^{-2 \mathrm{i} k z}\right\}\right] \\
& \frac{B_{0} j_{y}(z, t)}{u_{\mathrm{A}} \rho \omega_{\mathrm{g}}}=\zeta \mathfrak{J}\left[\mathrm{e}^{\mathrm{i} k\left(z-u_{\mathrm{A}} t\right)}\left\{1-\zeta_{1} \mathrm{e}^{-\mathrm{i} \Delta k z}-\zeta_{2} \mathrm{e}^{-2 \mathrm{i} k z}\right\}\right] \\
& -\frac{B_{x}(z, t)}{B_{0}}=\zeta \mathfrak{R}\left[\mathrm{e}^{\mathrm{i} k\left(z-u_{\mathrm{A}} t\right)}\left\{1-\zeta_{3} \mathrm{e}^{-\mathrm{i} \Delta k z}+\zeta_{2} \mathrm{e}^{-2 \mathrm{i} k z}\right\}\right] \\
& -\frac{\mu(z, t)}{\rho}=\zeta \mathfrak{R}\left[\mathrm{e}^{\mathrm{i} k\left(z-u_{\mathrm{A}} t\right)}\left\{1-\zeta_{4} \mathrm{e}^{-\mathrm{i} \Delta k z}+\zeta_{2} \mathrm{e}^{-2 \mathrm{i} k z}\right\}\right]
\end{aligned}
$$

with $k u_{\mathrm{A}}=\omega_{\mathrm{g}}, \Delta k=k-\omega_{\mathrm{g}}, \xi \equiv u_{\mathrm{A}} /\left(1+u_{\mathrm{A}}\right)^{2}$ and:

$$
\begin{array}{ll}
\zeta=\frac{h}{4} \zeta_{2}^{-\frac{1}{2}}, \zeta_{1}=4 \xi & \gg \zeta_{2}=u_{\mathrm{A}} \xi\left(\frac{\Delta k}{\omega_{\mathrm{g}}}\right)^{2}, \\
\zeta_{3}=4 u_{\mathrm{A}} \xi, \zeta_{4}=2 \xi \frac{1+u_{\mathrm{A}}^{2}}{u_{\mathrm{A}}} \gg \zeta_{2} .
\end{array}
$$

Since $\Delta k$ is very small (see Sect. 6.3), we can expand the solutions around $\Delta k=0$, or equivalently $u_{\mathrm{A}}=1$, to find the dominant terms:

$$
\begin{aligned}
\frac{B_{x}(z, t)}{B_{0}} & =\frac{v_{z}(z, t)}{u_{\mathrm{A}}}=\frac{\mu(z, t)}{\rho}=-\frac{E_{y}(z, t)}{u_{\mathrm{A}} B_{0}} \\
& \simeq \frac{h}{2} k z \mathfrak{J}\left[\mathrm{e}^{\mathrm{i} k\left(z-u_{\mathrm{A}} t\right)}\right], \\
\frac{B_{0} j_{y}(z, t)}{u_{\mathrm{A}} \rho \omega_{g}} & \simeq \frac{h}{2} k z \mathfrak{R}\left[\mathrm{e}^{\mathrm{i} k\left(z-u_{\mathrm{A}} t\right)}\right] .
\end{aligned}
$$

These are fast magneto-acoustic waves, propagating at the Alfvén speed in the same direction as the GW and growing linearly with distance.

\section{Observer frame}

For a plasma flowing out relativistically in the $z$-direction with Lorentz factor $\gamma_{\mathrm{tot}}=1 / \sqrt{1-\left(\beta+v_{z}\right)^{2}} \simeq \gamma+\gamma^{3} \beta v_{z}\left(\gamma \equiv \gamma_{\beta}\right)$ corresponding to a constant velocity $\beta \approx 1$, the full set of linearized GRM equations is:

$$
\begin{array}{rlr}
\left\{\frac{\partial}{\partial t}+\beta \frac{\partial}{\partial z}\right\} \mu & =-\gamma^{2} \rho\left\{\beta \frac{\partial}{\partial t}+\frac{\partial}{\partial z}\right\} v_{z} & \text { CONTINUITY } \\
\gamma^{3} \rho\left\{\frac{\partial}{\partial t}+\beta \frac{\partial}{\partial z}\right\} v_{z} & =-j_{y} B_{0} \\
\frac{\partial E_{y}}{\partial t}-\frac{\partial B_{z}}{\partial z}+4 \pi j_{y} & =\frac{\mathrm{i} \omega_{\mathrm{g}} h}{2}\left(B_{0}+E_{0}\right) \mathrm{e}^{\mathrm{i} \omega_{\mathrm{g}}(z-t)} & \text { AMPERE } \\
\frac{\partial E_{y}}{\partial z}-\frac{\partial B_{z}}{\partial t} & =\frac{\mathrm{i} \omega_{\mathrm{g}} h}{2}\left(B_{0}+E_{0}\right) \mathrm{e}^{\mathrm{i} \omega_{\mathrm{g}}(z-t)} & \text { FARADAY } \\
E_{y}+v_{z} B_{0}+\beta B_{x} & =0 \quad, E_{0}=-\beta B_{0}
\end{array}
$$

where all quantities are now defined in the observer frame at rest with respect to the binary (primes are used to indicate comoving quantities only where there is the risk of confusion). Explicitly: $\rho, \beta, B_{0}$ and $E_{0}=E_{y}^{(0)}$ are the zeroth order quantities and $\mu, v_{z}, B_{x}, E_{y}, j_{y}, h$ are the perturbations. The most important difference with respect to the comoving frame is the factor $(1-\beta) B_{0}$ in the $\mathrm{GW}$ terms, due to the background electric field seen by the observer. 
The solutions to the equilibrium system without the GWterms, are again $\propto \mathrm{e}^{\mathrm{i} \omega_{\mathrm{g}}\left(z / u_{\mathrm{A}}-t\right)}$ with $u_{\mathrm{A}}=\frac{\beta+u_{\mathrm{A}}^{\prime}}{1+\beta u_{\mathrm{A}}^{\prime}}$ the Lorentz boosted, generalized Alfvén speed. The full system can be solved along the same lines as in Sect. 4.2 by straightforward calculation using Laplace and Fourier transformations. The physical differences between the comoving and the observer frames are clear from Eqs. (17).

A different approach - leading to the same result - is to Lorentz transform Eqs. (14). Since Lorentz transformations are linear transformations and furthermore the phase of a plane wave is an invariant, the general solutions have the same form as Eqs. (14), but with different amplitudes.

The MSW components excited by a GW propagating through a relativistically flowing plasma are given in terms of observer quantities by:

$$
\begin{aligned}
\frac{E_{y}(z, t)}{u_{\mathrm{A}} B_{0}} & =\Lambda \mathfrak{R}\left[\mathrm{e}^{\mathrm{i} k\left(z-u_{\mathrm{A}} t\right)}\left\{1-\Lambda_{1} \mathrm{e}^{-\mathrm{i} \Delta k z}-\Lambda_{2} \mathrm{e}^{-2 \mathrm{i} k z}\right\}\right] \\
-\frac{B_{x}(z, t)}{B_{0}} & =\Lambda \mathfrak{R}\left[\mathrm{e}^{\mathrm{i} k\left(z-u_{\mathrm{A}} t\right)}\left\{1-\Lambda_{3} \mathrm{e}^{-\mathrm{i} \Delta k z}-\Lambda_{4} \mathrm{e}^{-2 \mathrm{i} k z}\right\}\right] \\
-\frac{v_{z}(z, t)}{u_{\mathrm{A}}-\beta} & =\Lambda \Re\left[\mathrm{e}^{\mathrm{i} k\left(z-u_{\mathrm{A}} t\right)}\left\{1-\Lambda_{5} \mathrm{e}^{-\mathrm{i} \Delta k z}-\Lambda_{6} \mathrm{e}^{-2 \mathrm{i} k z}\right\}\right] \\
\frac{B_{0} j_{y}(z, t)}{u_{\mathrm{A}} \rho \omega_{\mathrm{g}}} & =\Lambda \Re\left[\mathrm{e}^{\mathrm{i} k\left(z-u_{\mathrm{A}} t\right)}\left\{1-\Lambda_{7} \mathrm{e}^{-\mathrm{i} \Delta k z}-\Lambda_{8} \mathrm{e}^{-2 \mathrm{i} \kappa z}\right\}\right] \\
-\frac{\mu(z, t)}{\rho} & =\lambda \mathfrak{R}\left[\mathrm{e}^{\mathrm{i} k\left(z-u_{\mathrm{A}} t\right)}\left\{1-\Lambda_{9} \mathrm{e}^{-\mathrm{i} \Delta k z}-\Lambda_{6} \mathrm{e}^{-2 \mathrm{i} \kappa z}\right\}\right]
\end{aligned}
$$

where the constants $\Lambda-\Lambda_{9}, \lambda$ are defined in Appendix A. As in Sect. 4.2, the dominant terms are found by expanding around $\Delta k=0$ (justified in Sect. 6.3):

$$
\begin{aligned}
\frac{B_{x}(z, t)}{B_{0}}=-\frac{E_{y}(z, t)}{u_{\mathrm{A}} B_{0}}=\frac{v_{z}(z, t)}{u_{\mathrm{A}}-\beta} & =\frac{\mu(z, t)}{\rho}= \\
\frac{(1-\beta) h}{2} k z \mathfrak{I}\left[\mathrm{e}^{\mathrm{i} k\left(z-u_{\mathrm{A}} t\right)}\right] & \simeq \frac{h}{4} \frac{k z}{\gamma^{2}} \mathfrak{J}\left[\mathrm{e}^{\mathrm{i} k\left(z-u_{\mathrm{A}} t\right)}\right], \\
\frac{B_{0} j_{y}(z, t)}{\hat{\rho} \omega_{\mathrm{g}} u_{\mathrm{A}}} & \simeq \frac{h}{4} \frac{k z}{\gamma^{2}} \mathfrak{R}\left[\mathrm{e}^{\mathrm{i} k\left(z-u_{\mathrm{A}} t\right)}\right] .
\end{aligned}
$$

where: $\hat{\rho} \equiv \frac{B_{0}^{2}}{4 \pi} \frac{1-u_{\mathrm{A}}^{2}}{u_{\mathrm{A}}^{2}}$. Apparently, the interaction is less efficient when the plasma is escaping relativistically, than when it is at rest with respect to the source of GWs.

As a final remark: Eq. (19) is equivalent to the result of an initial value approach, where the Laplace transformations are performed with respect to $t$ instead of $z$. The amplitudes in Eq. (19) become proportional to $\omega t$ instead of $k z$, but the characteristic timescale $T$ is related to the size of the interaction region $R$ by: $\omega T \sim \omega\left(R / u_{\mathrm{A}}\right)=k R$.

\section{Numerical estimates}

In this section the results obtained in the previous sections are applied to the environs of a NS-NS binary close to merging. Both stars will have an electron-positron wind filling the surrounding space with plasma up to large distances (whether the plasma is $e^{ \pm}$or baryon loaded is not important as long as it satisfies the ideal MHD condition). The extent of the interaction region is determined by the distance $R_{\max }$ from the source where either the force-free or the $z \ll 1 / \Delta k$ assumption brakes down. Within this scale height, we estimate the magnitude of the excited MSW amplitudes, including the decrease of the GWamplitude, the density and the magnetic field with distance. A short numerical analysis is made for the magnetic fields, plasma densities, Lorentz factors and Alfvén velocities in the relativistic plasma wind, summarized in Table 1.

Note: In this section proper dimensions in $c$ are restored and the numerical results are converted to SI units.

\subsection{Magnetic field configuration}

For pulsar-like neutronstars, the magnetic field close to the surface falls of as a dipole: $B(r)=B_{\star}\left(R_{\star} / r\right)^{3}$. In the MHD approximation the plasma is "glued" to the field lines and forced to corotate up to the lightcylinder where corotation requires superluminal velocities. Here, we consider a NS-NS merger where each NS has its own magnetic field. The key element in the electromagnetic description of rotating magnetized stars is the deviation from inertial motion. In our case this is not the usual stellar rotation but the orbital motion combined with the individual stellar rotations. As the binary coalesces the orbital frequency increases and dominates over any other (rotational) motion. Therefore, we assume that at the end of the spiral-in phase the orbital rotation of the binary (with $\Omega_{\mathrm{b}} \sim 10^{3} \mathrm{rad} / \mathrm{s}$ ) determines the light-cylinder radius: $R_{\mathrm{lc}}=c / \Omega_{\mathrm{b}} \simeq 300 \mathrm{~km}$. Here the field lines, anchored on the stellar polar caps, open up and the plasma is free to flow out along the field in a force-free wind in which the toroidal component of the field dominates the poloidal component (Kuijpers 2001):

$$
\begin{aligned}
& B_{\mathrm{t}}\left(r>R_{\mathrm{lc}}\right)=B_{\mathrm{lc}}\left(\frac{R_{\mathrm{lc}}}{r}\right), \quad B_{\mathrm{p}}\left(r>R_{\mathrm{lc}}\right)=B_{\mathrm{lc}}\left(\frac{R_{\mathrm{lc}}}{r}\right)^{2}, \\
& B_{\mathrm{lc}}=B_{\star}\left(\frac{\Omega_{\mathrm{b}} R_{\star}}{c}\right)^{3} \simeq 4 \times 10^{5} \mathrm{~T}\left(B_{\star, 9}\right)\left(\Omega_{\mathrm{b}, 3}\right)^{3}\left(R_{\star, 4}\right)^{3} .
\end{aligned}
$$

\subsection{Nature of the wind}

Above the polar caps of a pulsar the field lines are open, particles flow outwards and a steady charge density cannot be maintained at the Goldreich-Julian density everywhere $n_{\mathrm{GJ}}=$ $\boldsymbol{\Omega}_{\mathrm{b}} \cdot \boldsymbol{B} /(2 \pi e c)$ (Beskin et al. 1993; Lyubarsky 1995). As a result, a strong electric field develops along the magnetic field above the polar cap and charged "primary" particles are extracted from the surface with a density $n_{\mathrm{p}} \simeq n_{\mathrm{GJ} \star}$ and accelerated to high Lorentz factors (a typical number is $\gamma_{\mathrm{p}} \sim 10^{7}$ ). Note that the available potential jump is proportional to $\Delta \Psi \propto \Omega_{\mathrm{b}} B_{\star}$ in our case and can be much larger than for single pulsars.

Processes such as curvature radiation and inverse Compton emission then result in a cascade of "secondary" $e^{ \pm}$pairs with a particle number density $n_{\mathrm{s}}=M n_{\mathrm{p}}$, where $M$ is called the multiplicity. Due to energy conservation $n_{\mathrm{p}} \gamma_{\mathrm{p}}=n_{\mathrm{s}} \gamma_{\mathrm{s}}$, so the Lorentz factor of the secondary particles is $\gamma_{\mathrm{s}}=\gamma_{\mathrm{p}} / M \sim 100$ for $M \sim 10^{5}$. The secondary plasma flows out as a relativistic wind along the open magnetic field lines which have a dipole-like radial dependence up to $R_{\mathrm{lc}}$, and develop into a spiral (Eq. (20)) further out. The charge density in the wind is adjusted to the 
Table 1. Formulas and numerical values in the frames at rest with respect to an observer and the plasma, respectively. In the numerical examples of this table we have taken: $B_{\star}=10^{9} \mathrm{~T}, \gamma=\gamma_{\mathrm{s}}=100, M=10^{5}, \Omega_{\mathrm{b}} / 2 \pi=160 \mathrm{~Hz}$.

\begin{tabular}{|c|c|c|c|}
\hline \multicolumn{2}{|c|}{ Lab/Observer frame } & \multicolumn{2}{|c|}{ Plasma/Comoving frame } \\
\hline $\begin{aligned} \omega_{\mathrm{g}} & =k_{\mathrm{g}} c \\
\omega_{\mathrm{MSW}} & =\omega_{\mathrm{g}}=k_{\mathrm{MSW}} u_{\mathrm{A}}\end{aligned}$ & $\begin{aligned} k_{\mathrm{g}} & \sim 2 \times 10^{-5} \mathrm{~m}^{-1} \\
\omega_{\mathrm{g}} & \sim 2 \pi \times 10^{3} \mathrm{rad} \mathrm{s}^{-1}\end{aligned}$ & $\begin{aligned} \omega_{\mathrm{g}}^{\prime} \approx \omega_{\mathrm{g}} /(2 \gamma) & =k_{\mathrm{g}}^{\prime} c \\
\omega_{\mathrm{MSW}}^{\prime}=\omega_{\mathrm{g}}^{\prime} & =k_{\mathrm{MSW}}^{\prime} u_{\mathrm{A}}^{\prime}\end{aligned}$ & $\begin{aligned} k_{\mathrm{g}}^{\prime} & \sim 10^{-7} \mathrm{~m}^{-1} \\
\omega_{\mathrm{g}}^{\prime} & \sim 30 \mathrm{rad} \mathrm{s}^{-1}\end{aligned}$ \\
\hline $\begin{array}{l}\rho_{\star}=\frac{M m_{\mathrm{e}}}{e}\left(\frac{\Omega_{\mathrm{b}} B_{\star}}{2 \pi c}\right) \\
\rho_{\mathrm{lc}}=\rho_{\star}\left(\frac{B_{\mathrm{lc}}}{B_{\star}}\right)\end{array}$ & $\begin{array}{l}\rho_{\mathrm{lc}} \sim 4 \times 10^{-10} \mathrm{~kg} \mathrm{~m}^{-3} \\
\Omega_{\mathrm{e}}=\frac{e B_{\mathrm{lc}}}{m_{\mathrm{ec}}} \sim 7 \times 10^{15} \mathrm{rad} \mathrm{s}^{-1}\end{array}$ & $\begin{aligned} \frac{v_{A}^{\prime 2}}{c^{2}}=\frac{B^{\prime 2}}{4 \pi \rho^{\prime} c^{2}} & =\frac{B_{1 c}^{2}}{4 \pi \gamma \rho_{\mathrm{c}} c^{2}} \\
& =\frac{\Omega_{\mathrm{e}}}{2 \gamma_{\mathrm{p}} \Omega_{\mathrm{b}}}\end{aligned}$ & $v_{\mathrm{A}}^{\prime} \sim 570 c$ \\
\hline $\begin{aligned} u_{\mathrm{A}} & =\frac{u_{\mathrm{A}}^{\prime}+\beta c}{1+\beta u_{\mathrm{A}}^{\prime} / c} \\
\gamma_{u_{\mathrm{A}}} & \approx 2 \gamma \gamma_{u_{\mathrm{A}}^{\prime}}\end{aligned}$ & $\gamma_{u_{\mathrm{A}}} \sim 10^{5}$ & $\begin{aligned} \frac{u_{A}^{\prime 2}}{c^{2}} & =\frac{v_{A}^{\prime 2}}{c^{2}+v_{A}^{\prime 2}} \\
\gamma_{u_{A}^{\prime}}^{2} & \approx \frac{\Omega_{e}}{2 \gamma_{p} \Omega_{b}}\end{aligned}$ & $\gamma_{u_{\mathrm{A}}^{\prime}} \sim 570$ \\
\hline $\begin{aligned} \Delta k & =\frac{\omega_{\mathrm{g}}}{c}\left(\frac{c}{u_{\mathrm{A}}}-1\right) \\
& =\frac{\Delta k^{\prime}}{2 \gamma}\end{aligned}$ & $\Delta k \sim 8 \times 10^{-16} \mathrm{~m}^{-1}$ & $\begin{aligned} \Delta k^{\prime} \equiv k^{\prime}-k_{\mathrm{g}}^{\prime} & =\frac{\omega_{\mathrm{g}}^{\prime}}{c}\left(\frac{c}{u_{\mathrm{A}}^{\prime}}-1\right) \\
& \approx \frac{\omega_{\mathrm{g}} M}{2 c} \frac{\Omega_{\mathrm{b}}}{\Omega_{\mathrm{e}}}\end{aligned}$ & $\Delta k^{\prime} \sim 1.6 \times 10^{-13} \mathrm{~m}^{-1}$ \\
\hline$R \Delta k \ll 1$ & $R_{\max } \sim \frac{0.075}{\Delta k} \sim 10^{14} \mathrm{~m}$ & & \\
\hline
\end{tabular}

local Goldreich-Julian density everywhere and the wind remains force-free up to a large distance.

At the lightcylinder the density is:

$$
n_{\mathrm{lc}}=n_{\star} \frac{B_{\mathrm{lc}}}{B_{\star}}=\frac{M \Omega_{\mathrm{b}} B_{\mathrm{lc}}}{2 \pi e c} \sim 4 \times 10^{20} \mathrm{~m}^{-3} .
$$

\subsection{Alfvén speeds and Interaction lengthscale}

The classical Alfvén speed $v_{\mathrm{A}}$ is proportional to $B_{0} / \sqrt{n}$. In the force free wind we have $B_{0} \propto 1 / r$ and $n \propto 1 / r^{2}$, so $v_{\mathrm{A}}\left(r>R_{\mathrm{lc}}\right)=$ const. Therefore, we can evaluate the Alfvén velocities at the light-cylinder in the comoving frame (using Eqs. (21)):

$$
\begin{aligned}
& B_{\mathrm{lc}}^{\prime}=\frac{B_{\mathrm{lc}}}{\gamma_{\mathrm{s}}} \quad \text { and } \quad n_{\mathrm{lc}}^{\prime}=\frac{n_{\mathrm{lc}}}{\gamma_{\mathrm{s}}}, \\
& \frac{v_{\mathrm{A}}^{\prime 2}}{c^{2}}=\frac{B_{\mathrm{lc}}^{\prime 2}}{4 \pi n_{\mathrm{lc}}^{\prime} m_{\mathrm{e}} c^{2}}=\frac{B_{\mathrm{lc}}^{2}}{4 \pi \gamma_{\mathrm{s}} n_{\mathrm{lc}} m_{\mathrm{e}} c^{2}}=\frac{\Omega_{\mathrm{e}}}{2 \gamma_{\mathrm{p}} \Omega_{\mathrm{b}}} \gg 1,
\end{aligned}
$$

in terms of the electron cyclotron frequency $\Omega_{\mathrm{e}}=\frac{e B_{\mathrm{l}}}{m_{\mathrm{e}} c}$. For the generalized Alfvén speed and the wavenumber difference $\Delta k^{\prime}$, one finds (see also Table 1):

$$
\begin{aligned}
& \frac{u_{\mathrm{A}}^{\prime 2}}{c^{2}}=\frac{v_{\mathrm{A}}^{\prime 2}}{c^{2}+v_{\mathrm{A}}^{\prime 2}} \simeq 1 \quad \text { or } \quad \gamma_{u_{\mathrm{A}}^{\prime}}^{2} \approx \frac{\Omega_{\mathrm{e}}}{2 \gamma_{\mathrm{p}} \Omega_{\mathrm{b}}} \gg 1, \\
& \Delta k^{\prime} \equiv k^{\prime}-k_{\mathrm{g}}^{\prime}=\frac{\omega_{\mathrm{g}}^{\prime}}{c}\left(\frac{c}{u_{\mathrm{A}}^{\prime}}-1\right) \ll 1 .
\end{aligned}
$$

The equivalent quantities in the observer frame are derived from Lorentz transformations and can be found in Table 1 . The important conclusion from these estimates is that the maximum distance over which the MSW amplitudes can grow is: $z \ll 1 / \Delta k \simeq 10^{15} \mathrm{~m}$ (see Eq. (19)), which is comparable to the limits on the MHD approximation as calculated by Spruit et al. (2001).

\subsection{Magnitude of excited magnetosonic waves}

The frequency of GWs emitted by a merging binary is related to the angular frequency of the last orbits by: $\omega_{\mathrm{g}}=2 \Omega_{\mathrm{b}} \sim 4 \pi \times$ $10^{3} \mathrm{rad} / \mathrm{s}$ (Shibata \& Uryu 2002), so $R_{\mathrm{lc}} \sim 50 \mathrm{~km}$ and $B_{\mathrm{lc}} \sim$ $10^{7} \mathrm{~T}$. We assume that for $R_{\text {in }} \sim 10 R_{\mathrm{lc}}$ we are in the far field of the merger and estimate the amplitude of $B_{x}^{(1)}(z, t)$ in Eq. (19) at the start of the interaction region. Taking into account the (approximate) $1 / r$ decrease of $h$ and $B_{0}$,

$h \sim 10^{-3}\left(\frac{R_{\text {in }}}{r}\right), \quad B_{0} \sim B_{\mathrm{lc}}\left(\frac{R_{\mathrm{lc}}}{R_{\text {in }}}\right)\left(\frac{R_{\mathrm{in}}}{r}\right)$,

we find:

$B^{(1)}\left(r>R_{\mathrm{in}}\right) \sim 0.5 \mathrm{~T}\left(\frac{R_{\mathrm{in}}}{r}\right) \times\left[\frac{h_{-3}}{\left(\gamma_{2}\right)^{2}} k_{\mathrm{g},-5} B_{\mathrm{lc}, 7} R_{\mathrm{in}, 5}\right]$.

The volume integrated energy of the MSW grows linearly with distance since $B^{(1)} \propto B_{0} \propto 1 / r$ and $V \propto r^{3}$. For an interaction region of $R_{\max } \sim 0.03 \mathrm{pc}$ we find for the magnetic component of the excited MHD wave a total energy of:

$\mathrm{T}_{\mathrm{B}}^{(1)}=V \frac{B_{0} B^{(1)}}{4 \pi} \sim 10^{37} \mathrm{~J}$,

which amounts to a fraction $10^{-6}$ of the magnetic energy $\mathrm{T}_{\mathrm{B}}^{(0)}=$ $\frac{V B_{0}^{2}}{8 \pi} \sim 10^{43} \mathrm{~J}$ of the Poynting flux dominated wind in the same volume.

\section{Discussion}

It is known that, to first order in $h$, GWs propagating along a uniform magnetic field do not couple to the field, neither in vacuum (Boccaletti et al. 1970), nor in a plasma (Brodin et al. 2000; Papadopoulos et al. 2001) so we studied GW-propagation perpendicular to the magnetic field frozen into a plasma. An intuitive illustration of the process is given in Fig. 1, where a GW 


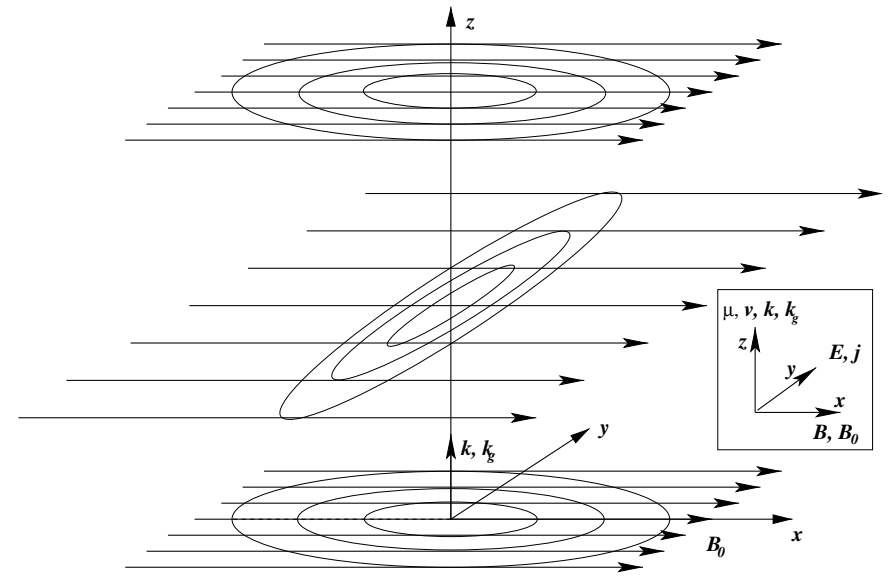

Fig. 1. A GW propagating in the positive $z$-direction across an ambient magnetic field (in the $x$-direction) excites a MSW. The orientations of the MSW components are indicated in the inset.

traveling in the positive $z$-direction, deforms (imaginary) rings of plasma-particles in the $x-y$ plane into ellipses with long axes alternating periodically along the magnetic field direction ( $x$ axis) and the $y$-axis. Consequently, the uniform field is periodically compressed and stretched leading to a modulated magnetic field strength even though the GW is divergence-free. The resulting magnetic pressure gradients in the $z$-direction try to re-establish a uniform field configuration and excite the compressional matter part of the MSW. Since the plasma is glued to the field lines it is dragged along with the field. The velocity in the $z$-direction generates an electric field $(-\boldsymbol{v} \times \boldsymbol{B})$ with a corresponding $\boldsymbol{E} \times \boldsymbol{B}$ drift-velocity, whereas the magnetic gradient induces a $\boldsymbol{B} \times \nabla \boldsymbol{B}$ current density and Lorentz force.

The problem of GW propagation in a plasma at rest was also studied by Papadopoulos et al. (2001) who found that the excitation of MSWs by a GW is only possible when there is a wavenumber mismatch: $B_{x}^{(1)} \propto \Delta k$. This does not agree with our results (as presented in Sect. 4.2), nor with those of Marklund et al. (2000) who show that the growth rate in a tenuous plasma smoothly matches that in a vacuum (Boccaletti et al. 1970). Both from the GRM equations (Eqs. (8)-(11) or (17)) and from their solutions (Eqs. (16) or (19)) it is clear that in the vacuum limit $\mu^{(1)} \propto j_{y}^{(1)} \propto \rho \downarrow 0$ vanish as does $v_{z}^{(1)}$. The GWs excite EMWs that propagate with the speed of light $(\Delta k \downarrow 0)$ and with amplitudes that can be obtained from Eq. (16) or Eq. (19) by taking the limit $u_{\mathrm{A}} \rightarrow c$ and $k \rightarrow k_{\mathrm{g}}$ (and $\gamma=1$ ). This result is not surprising as we have taken into account both the material current and the vacuum displacement current.

\section{Conclusion}

The results presented in this paper are a first attempt to study the interaction of the relatively strong GWs emitted by a GRB with an ultra-relativistic plasma wind. The space surrounding a merging NS-NS binary is already filled with such a wind up to large distances $(\sim 0.1 \mathrm{pc})$. Moreover, in the merger almost all of the binding energy is released in the form of GWs.

We derive a closed set of GRM equations both in the natural orthonormal measurement frame (the $3+1$ split) of the plasma and for an observer at rest with respect to the binary. These noncoordinate equations strongly resemble their Newtonian equivalents but have extra source terms due to the GW. These gravity terms act as a driver for fast magnetosonic waves with amplitudes that grow linearly with distance and are proportional to the GW-frequency and amplitude and to the ambient magnetic field strength. It is the extended force-free wind in which the magnetic field only falls of as $1 / r$ that provides the long interaction lengthscale.

The total amount of energy that is transferred from the GWs to the plasma, as given in Eq. (25), is substantial but for this case still much smaller than the average observed GRB energy. Note that for magnetars one can have $B_{\star} \sim 10^{12} \mathrm{~T}$ and $\omega_{\mathrm{g}} \sim$ $15 \mathrm{kHz}$, so that $\left(B_{0} B^{(1)}\right) / 4 \pi$ could be as much as a factor $10^{7}$ larger. However, for magnetars it is not obvious what to assume for the surrounding plasma.

In future work we will investigate what the observable effects of the GW-MSW interaction are on the emitted radiation and its polarization and how a GW-chirp alters the results.

Acknowledgements. We would like to thank V. S. Beskin, D. Papadopoulos, R. Pudritz, N. Stergioulas and L. Vlahos for useful discussions. This work was supported by the Dutch Research School for Astronomy, NOVA.

\section{Appendix A: Constants in Eq. (18)}

$$
\begin{aligned}
& \Lambda=\frac{h}{4} \frac{1+u_{\mathrm{A}}}{1-u_{\mathrm{A}}} \frac{(1-\beta)^{2}}{1-u_{\mathrm{A}} \beta} \simeq \frac{h}{4} \frac{\omega_{\mathrm{g}}}{\gamma^{2} \Delta k} \\
& \Lambda_{1}=2 \frac{1-u_{\mathrm{A}} \beta}{(1-\beta)^{3}} \frac{\beta\left(1-4 u_{\mathrm{A}}+\beta\right)+u_{\mathrm{A}}^{2}(2-\beta(1-\beta))}{u_{\mathrm{A}}\left(1+u_{\mathrm{A}}\right)^{2}}=1+O[\Delta k]^{2} \\
& \Lambda_{2}=\gamma^{2}\left(\frac{1-u_{\mathrm{A}}}{1+u_{\mathrm{A}}} \frac{1+\beta}{1-\beta}\right)^{2} \frac{u_{\mathrm{A}}-2 \beta+u_{\mathrm{A}} \beta^{2}}{u_{\mathrm{A}}}=O[\Delta k]^{2} \\
& \Lambda_{3}=2 \frac{1-u_{\mathrm{A}} \beta}{(1-\beta)^{3}} \frac{\left(1+u_{\mathrm{A}}^{2}+2 \beta^{2}-\left(1+\left(4-u_{\mathrm{A}}\right) u_{\mathrm{A}}\right) \beta\right.}{\left(1+u_{\mathrm{A}}\right)^{2}}=1+O[\Delta k]^{2} \\
& \Lambda_{4}=-\gamma^{2}\left(\frac{1-u_{\mathrm{A}}}{1+u_{\mathrm{A}}} \frac{1+\beta}{1-\beta}\right)^{2}\left(\beta^{2}-2 u_{\mathrm{A}} \beta+1\right)=O[\Delta k]^{2} \\
& \Lambda_{5}=4 \frac{u_{\mathrm{A}}-\beta}{\left(1+u_{\mathrm{A}}\right)^{2}} \frac{1-u_{\mathrm{A}} \beta}{(1-\beta)^{2}}=1-O[\Delta k]^{2}
\end{aligned}
$$$$
\Lambda_{6}=\left(\frac{1-u_{\mathrm{A}}}{1+u_{\mathrm{A}}} \frac{1+\beta}{1-\beta}\right)^{2}=O[\Delta k]^{2}
$$$$
\Lambda_{7}=\frac{4 u_{\mathrm{A}}}{\left(1+u_{\mathrm{A}}\right)^{2}} \frac{1+u_{\mathrm{A}} \beta}{1-\beta}=1+O[\Delta k]
$$$$
\Lambda_{8}=\left(\frac{1+u_{\mathrm{A}}}{1-u_{\mathrm{A}}}\right)^{2} \frac{\gamma^{2} u_{\mathrm{A}}(1+\beta)^{4}}{u_{\mathrm{A}}-2 \beta+u_{\mathrm{A}} \beta^{2}}=O[\Delta k]^{2}
$$$$
\Lambda_{9}=4 \gamma^{2}\left(\frac{u_{\mathrm{A}}-\beta}{1+u_{\mathrm{A}}}\right)^{2}=1+O[\Delta k]
$$ 
$\lambda=\frac{h}{4} \frac{1+u_{\mathrm{A}}}{1-u_{\mathrm{A}}} \frac{1-\beta}{1+\beta}=\gamma^{2}\left(1-u_{\mathrm{A}} \beta\right) \Lambda$

$\kappa=-\frac{\omega_{\mathrm{g}}}{u_{\mathrm{A}}} \frac{u_{\mathrm{A}}\left(1+\beta^{2}\right)-\beta\left(1+u_{\mathrm{A}}^{2}\right)}{u_{\mathrm{A}}\left(1+\beta^{2}\right)-2 \beta} \simeq \frac{\omega_{\mathrm{g}}}{u_{\mathrm{A}}}(1+O[\Delta k])$.

\section{References}

Beskin, V. S., Gurevich, A. V., \& Istomin, Y. N. 1993, Physics of the pulsar magnetosphere (Cambridge University Press)

Boccaletti, D., de Sabbata, V., Fortini, P., \& Gualdi, C. 1970, Il Nuovo Cimento, 70B, 129

Brodin, G., \& Marklund, M. 1999, Phys. Rev. Lett., 82, 3012

Brodin, G., Marklund, M., \& Dunsby, P. K. S. 2000, Phys. Rev. D., 62, 104008

Brodin, G., Marklund, M., \& Dunsby, P. K. S. 2001a, Classical and Quantum Gravity, 18, 5249

Brodin, G., Marklund, M., \& Servin, M. 2001b, Phys. Rev. D., 63, 124003

Ellis, G. F. R., \& van Elst, H. 1999, in Theoretical and Observational Cosmology, ed. M. Lachiéze-Rey (Dordrecht: Kluwer), 1-116, preprint http://arxiv.org/abs/gr-qc/9812046

Gerlach, U. H. 1974, Phys. Rev. Lett., 32, Nr. 18

Gertsenshtein, M. 1961, Zh. Exsp. Teor. Fiz., 41, 113

Hawking, S. W., \& Ellis, G. F. R. 1973, The large scale structure of space-time, Cambridge Monographs on Mathematical Physics (London: Cambridge University Press)
Janka, H. T., Eberl, T., Ruffert, M., \& Fryer, C. L. 2002

Kuijpers, J. 2001, Publ. Astron. Soc. Austr., 18, 407

Landau, L. D., \& Lifshitz, E. M. 1975, The classical theory of fields, Course of theoretical physics - Pergamon International Library of Science, Technology, Engineering and Social Studies (Oxford: Pergamon Press), 4th rev. engl. ed.

Lichnerowicz, A. 1967, Relativistic Hydrodynamics and Magnetohydrodynamics (New York: Benjamin)

Lupanov, G. A. 1967, Sov. Phys. JETP, 25, 76

Lyubarsky, Y. E. 1995, Physics of pulsars (Amsterdam: Harwood Academic Publishers)

Macedo, P. G., \& Nelson, A. H. 1983, Phys. Rev. D., 28, 2382

Marklund, M., Brodin, G., \& Dunsby, P. K. S. 2000, ApJ, 536, 875

Melrose, D. B. 1986, Instabilities in space and laboratory plasmas (Cambridge and New York: Cambridge University Press), 290

Misner, C. W., Thorne, K. S., \& Wheeler, J. A. 1973, Gravitation (San Francisco: W.H. Freeman and Co.)

Papadopoulos, D., Stergioulas, N., Vlahos, L., \& Kuijpers, J. 2001, A\&A, 377, 701

Shibata, M., \& Uryu, K. 2002, Prog. Theor. Phys., 107, 265

Spruit, H. C., Daigne, F., \& Drenkhahn, G. 2001, A\&A, 369, 694

Thorne, K. S., Price, R. H., \& MacDonald, D. A. (eds.) 1986, Black holes: The membrane paradigm

Weinberg, S. 1972, Gravitation and cosmology: Principles and applications of the general theory of relativity (New York: Wiley)

Zel'dovich, Y. B. 1973, Zh. Eksp. Teor. Fiz., 65, 1311 